\title{
Region-wise Ranking for One-Day International (ODI) Cricket Teams
}

\author{
Akbar Hussain $^{1}$, Yan Qiang*,2 ${ }^{*}$ M. Abdul Qadoos Bilal ${ }^{3}, \mathrm{Kun} \mathrm{Wu}^{4}$, Zijuan Zhao ${ }^{5}$, Bilal Ahmed ${ }^{6}$ \\ College of Information and Computer \\ Taiyuan University of Technology, Taiyuan, Shanxi, China
}

\begin{abstract}
In cricket, the region plays a significant role in ranking teams. The International Cricket Council (ICC) uses an ad-hoc points system to rank cricket teams, which entirely based on the number of wins and losses a match. The ICC ignores the strength and weaknesses of the team across the region. Even though the relative accuracy in the ad-hoc ranking is high, but they do not provide a clearly defined method of ranking. We proposed Region-wise Team Rank (RTR) and a Region-wise Weighted Team Rank (RWTR) to rank cricket teams. The intuition is to get more points to a team that wins a match from a stronger team as compared to a team that wins against a weaker team \& vice versa. The proposed method considers not only the number of region-wise wins and losses but also incorporates the region-wise strength and weakness of a team while assigning them the ranking score. In conclusion, the ranking list of the teams compares to the ICC official ranking.
\end{abstract}

Keywords---Batting; bowling and fielding strength; PageRank; region strength; team's strength

\section{INTRODUCTION}

Ranking in sports is a significant measure for the performance and comparison between different teams. The International Cricket Council (ICC) is the governing body that ranks cricket players and teams based on their previous and current performances. Traditionally, the ICC uses an ad-hoc rating system to rank cricket teams. This Ad-hoc rating system is based on the number of wins or loses in a match to assign a rating score 1 . Authors criticized the current ICC rating system as being non-transparent, and providing a better ranking still needs proper investigation [1].

There are five region in the world in which cricket is played. These regions are classified as follows; Asia, Africa, the Americas, Europe, and Oceania Region ${ }^{2}$. In cricket, the region/venue plays an essential role in measuring players' and teams 'performance because every region has distinct playing conditions. Region-wise ranking classifies the team's strengths and weaknesses throughout the region/venue. The team's strength is the foundation of several parameters of a team, such as bowling, batting, and fielding strengths. The consistent performance of the players in these departments improves their team's strength. The teams with higher strength are usually considered higher in rank. Ahmed et al. proposed the modified NSGA-II procedure to find a set of high-

\footnotetext{
*Corresponding Authors

${ }^{1} \mathrm{https}: / / \mathrm{www}$.sportsrec.com/calculate-followon-score-cricket8088680.html

${ }^{2} \mathrm{https}: / / \mathrm{www}$.icc-cricket.com/about/members
}

performing teams having better bowling and batting performance [2].

The proposed methods are the extensions of the PageRank Algorithm [3]. PageRank is a ranking algorithm that models the searchable web pages as a directed graph, with each page acting as a node. Every webpage has several numbers of incoming and outgoing links or links directed to that webpage. These links are "citations" concerning web pages. The idea is that the more a team wins matches from stronger teams, the higher it is ranked. The Region-wise Team Rank (RTR) considers the strengths and weaknesses and as well as win and loss matches of the team while ignores the number of runs and wickets from which matches wins. Besides, the Region-wise Weighted Team Rank (RWTR), considered both the strength of the team and the number of runs and wickets through which matches wins. Finally, the region-wise score of all the regions of the RTR method combined and computed the Unified Region-based Team Rank (URTR). Similarly, the Regionwise Weighted Team Rank (RWTR) scores merged and computed the Unified Region-wise Weighted Team Rank (RWTR). In conclusion, both the URTR and RWTR scores are merged and compared with the ICC official ranking using Spearmans' rho.

\section{RELATED WORK}

Research has identified a limited material set of references regarding region-wise and a unified team's strength; however, a comprehensive review revealed the following. The author developed a new performance measure to quantify the performance of the players [4]. A player scoring runs against a strong bowling lineup or taking wickets against the strong batting lineup deserves more credit. Batting, bowling, and fielding have always been the most critical aspects of the cricket game. But with the advent of T20 cricket, the combined batting and bowling rate quantify the performance of the player using multiple linear regressions [11]. The multiple linear regression model used [9] to predict the match outcome, while the match is in progress. Different variables are considered for training and testing the model, such as home ground advantage, region performance, the past and current form of the team to predict the match outcome. Logistic regression is applied to predict the best team after the knock-out phase in the ODI cricket series [6]. Fielding is also an essential aspect of the cricket game. Parag Shah [7] has developed to measure the aggregated fielding performance of each player in ODI and T20 cricket matches. The application of the Social Network Analysis (SNA) quantifies the quality of the player-versus-player score [5]. The application of 
machine learning framework, the artificial neural network (ANN) is applied to predict the results of cricket sports [8]. The simulation method is applied for ODI cricket matches to predict the match outcome. Given that only a finite number of outcomes can occur, throwing each ball. A discrete generator on a finite set developed where the outcome probabilities estimated from historical data of the matches [10].

A team that wins a match through runs and wickets plays a significant role in the team's ranking. Similarly, weighting factors are also an essential parameter in ranking cricket teams. Daud et al. [12] proposed team rank and t-index to rank cricket teams, but they do not consider the strengths and weaknesses of the teams across the region. The International Cricket Council (ICC) ranks cricket teams without considering the strengths and weaknesses across the region. However, Daud et al. [13] proposed a Region-based Players Link fusion (RPLF) algorithm to rank cricket players by using a regionbased intra-type and inter-type relation-based features to rank the players in cricket.

Page et al. develop PageRank for ranking web pages. Several researchers have attempted to improve the PageRank process [3]. Xing and Ghorbani proposed a weighted Pagerank (WPR) algorithm. The idea is to treat all links equally when rank scores calculated. The WPR algorithm takes into account the importance of both incoming and outgoing links and distributes rank scores among accessible web pages [14]. Many researchers in sports ranking used PageRank. The initial attempt was made by Mukherjee to apply the PageRank algorithm to identify the most excellent team and their captains [15]. Later on, the authors used PageRank for ranking cricket teams, but they ignored region-wise teams' strength and unified team strength, which is very important for the selection of the best team [12].

This research is probably the first generalized approach to rank ODI cricket teams across the region. The information retrieval technique is applied to build a model that can effectively measure the performance of a team across the region.

\section{RANKING CRICKET TEAMS}

The International Cricket Council (ICC) official ratings system for One Day International Cricket Teams explained in this section.

\section{A. ICC Rating System for ODI Cricket Teams}

The International Cricket Council (ICC) is the governing body to represent cricket internationally. They award championship trophies to the teams with the highest rating in cricket matches. The ICC employs basic formulas for ODI cricket matches to calculate points and ratings to each team by winning, losing, or tying a match or a series of matches ${ }^{[1]}$.

The ICC updates the ratings based on the recent score of the teams when two teams play a match or a series of matches. To determine the teams' new ratings after a particular match, first, calculate the points earned from the match.

- If the rating gap between two teams before the match is less than 40 points, then add 50 points more for a winning team than the opponent team's rating and assign 50 points fewer than the opponent's rating for losing a team. In case of a tie a match, each team assigns the opponent team's rating.

- If the gap between two teams' rating is greater than or equal to 40 points, then add 10 points more to a stronger team than its rating in case of winning a match or 90 points fewer than its rating by losing a match. Similarly, assign 90 points more to the total of a weaker team's rating for a win or 10 points fewer for a loss. In the case of a tie, the stronger team drops 40 points from its rating, and the weaker team improves 40 points over its original rating.

- Each team's rating is equal to its total points scored divided by the total matches played.

- Add the match points scored to the points already scored (in previous matches), add one to the number of matches played, and determine the new rating.

- Points earned by teams depend on the opponent's ratings. Therefore this system needed to assign base ratings to teams when it started started1.

\section{B. PageRank Algorithm}

Page et al. proposed a ranking algorithm named PageRank [3], which is used to rank web pages on the web. The PR of the pages should be high if they link to a more significant number of pages, and those pages are essential. PageRank based on the number of incoming and outgoing links of the pages (nodes) to determine their rank. Web pages with a higher number of incoming links are more important than pages with less number of incoming links. In general, the PageRank value for a web page determined by using the generalized equation as follows:

$P R(A)=c \sum_{v \in B_{A}} \frac{P R(v)}{L(v)}$

Where $\mathrm{PR}(\mathrm{A})$ is the value of PageRank of a page, A for each page $\mathbf{v}$ contained in the set $\mathrm{B}_{\mathrm{A}}$ (the set containing all the pages linking to page A), divided by the number of out-links $(\boldsymbol{L}(\boldsymbol{v}))$ from page $\mathbf{v}$ and $\mathbf{c}$ is a normalization factor.

\section{Cricket Formation and Terminologies}

1) Team formation: In cricket team selection, the batsman, bowler, all-rounder, and wicketkeeper plays a significant role in a match. Batsman scoring runs with a bat while a bowler bowls to concede minimum run and get maximum wickets. All-rounder can be a bowler or/and batter, and wicketkeeper is a player who stands behind the wickets to stop the ball or take a catch of a batsman.

2) Ground: The ground is usually in a circle-shaped having radius 70 meters. Different grounds in the world have a size of 70 meters or more. There is a 22 -meter pitch inside the ground having wickets on both sides. The bowler bowl and the batsmen try to hit a ball.

3) Over: An "over" is defined as a set of six consecutive balls bowled by a bowler.

4) Wicket: In cricket, the wicket is one of the two sets of three stumps at either side of the pitch. Wicket guarded a 
batsman who, with his bat, attempts to prevent the ball from hitting the wicket by a bowler or fielder.

5) Runs Scored: In cricket sports, one team bat first and score as many runs as possible while the other team bowls. In case of the team batting first wins, it wins by runs while in case team batting second wins, it wins by wickets. In the ODI cricket match, five bowlers have permission to bowl a maximum of 10 overs. The bowler bowls while batsmen try to hit a ball and get runs. Only two batsmen are playing in a pitch at the same time, one at each side. There is a maximum of 6 runs or no runs on a ball hit by a batsman. When a batsman hits a ball, it crosses a boundary without touching a boundary line; it should be considered a 6 runs otherwise 4 runs. When a batsman hits a ball and reaches to the other side of the pitch, he/she completes his one run.

\section{Proposed Method}

1) Region-wise team 'strength: In cricket sports, ranking is essential to measure the performance and comparison between different teams. Bowling, batting, and fielding performances are the most critical parameters for the team's strength. Players showing consistent performance in each of the departments improve their team strength.

We created three datasets from the online website 3 using scraping tools (parsehub and import.in) then created different features for each of the datasets of batting, bowling and fielding respectively. As a result, we combined the strengths of all the regions and find out the aggregated strength as shown in Fig. 1.

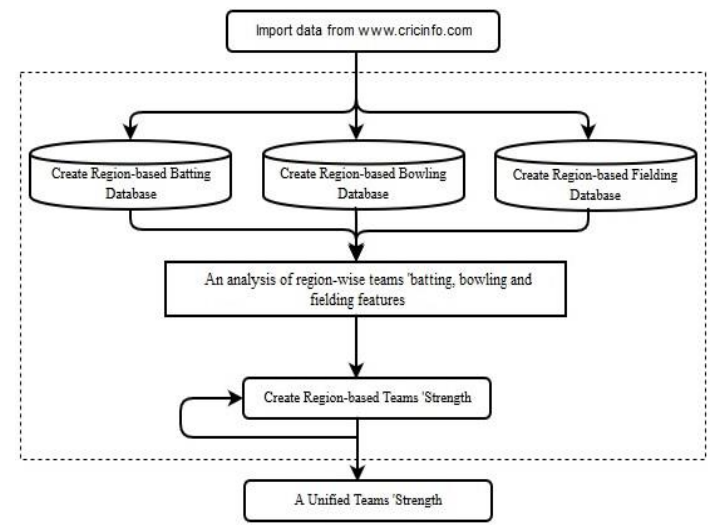

Fig. 1. A Graphical Representation of Region-based Teams' strength.

Equation (2) is applied to compute the region-wise teams 'strength.

$$
\begin{aligned}
& \sum_{t, r=i}^{n} R T_{\text {Strength }}= \\
& \quad \sum_{t, r=i}^{n}\left(\frac{\boldsymbol{R T}_{\text {Batting }_{\text {Strength }}}}{\boldsymbol{R T _ { \text { bowling } }}} * \boldsymbol{R} \boldsymbol{T}_{\text {Strength }}\right.
\end{aligned}
$$

Where $\sum_{t, r=i}^{n} R T_{\text {Strength }}$ is the region-wise strength of a team $\mathrm{t}$ in region $\mathrm{r}, R T_{\text {Batting_Strength }}$ is the batting strength, $R T_{\text {Bowling_Strength is the bowling strength and }}$ $R T_{\text {Fielding_Strength }}$ is the region-wise fielding strength. The team's strength increases with the increases of batting features while the team's strength decreases with the increases of bowling features.

Batsmen always perform against the opponent team's fielding and bowling strengths. The Team_AvgRun (team average runs), Team_ER (team economy rate), Team_SR (team strike rate), and Team_WinLoss (team win/loss ratio) is considered to be the most important parameters for the teams 'batting strength. These features are ascertained as in (3).

$\sum_{t, r=i}^{n} R T_{\text {Batting }}$ Strength $=$

$\sum_{t, r=i}^{n}\left(\frac{\left(\text { Team }_{\text {AvgRuns }} * \alpha\right)+\left(\text { Team }_{\mathrm{ER}} * \beta\right)+\left(\text { Team }_{\left.\mathrm{SR}^{*} \gamma\right)+(\text { Team_WinLos } * \delta}\right.}{4}\right)$

Where $\sum_{t, r=i}^{n} R T_{\text {Batting_Strength }}$ is the region-wise batting strength of team $\mathrm{t}$ in region $\mathrm{r}$., and $\alpha+\beta+\gamma+\delta=1$, finding the optimum combination of these variables is difficult in the absence of gold standard benchmark rankings. Daud et al. assigned $\alpha, \beta, \gamma$ and $\delta$ to different weights such as $\alpha=$ $\beta=\gamma=20 \%$ and $\delta=60 \%$; however, we used $\alpha=\beta=\gamma=$ $\delta=25 \%$ to each of the features [13], [12].

Many features affect the bowling strength of a team. The essential features are applied to compute the accurate bowling strength of a team in (4).

$\sum_{t, r=i}^{n} R T_{\text {Bowling }}$ Strength $=$

$\sum_{t, r=i}^{n}\left(\frac{(\text { AvgRuns_Conceded } * \alpha)+(\text { ER_Conceded } * \beta)+(\text { SR_Conceded } * \gamma)+(\text { Team_WLLoss } * \delta)}{4}\right)$

Where $\sum_{t, r=i}^{n} R T_{\text {Bowling_Strength }}$ is the region-wise bowling strength of team $t$ in region r. AvgRuns_Conceded, ER_Conceded, SR_Conceded and Team_WinLoss are the region-wise team's bowling average runs conceded, bowling economy rate conceded, bowling strike rate conceded and teams 'win/loss ratio respectively.

Several features are affecting the fielding performance of the team. The essential features are formulated in (5) to compute the fielding strength of the teams across the region.

$\sum_{t, r=i}^{n} R T_{\text {Fielding_Strength }}=$

$\sum_{t, r=i}^{n}\left(\frac{\left(\mathrm{RT}_{\text {run_out }} * \alpha\right)+\left(\mathrm{RT}_{\text {wicket_stmp }} * \beta\right)+\left(\mathrm{RT}_{\text {fielder_cats }} * \gamma\right)+\left(\mathrm{RT}_{\text {wicket_keeper_cats }} * \delta\right)}{\text { Total Number } \text { of Region_based Innings Played }}\right)$

Where $\sum_{t, r=i}^{n} R T_{\text {Fielding_Strength }}$ is the region-wise fielding strength of team $t$ in region $r, R_{\text {run_out, }} \mathrm{RT}_{\text {wicket_stmp }}$, $\mathrm{RT}_{\text {fielder_cats }}$, and $\mathrm{RT}_{\text {wkpr_cats }}$ are the number of region-based runout, wicketkeeper stumps, fielder catches and wicketkeeper catches of team $t$ in region $r$ respectively.

2) Region-wise TeamRank (RTR): The Region-wise TeamRank (RTR) is the extension of the PageRank algorithm. PageRank [3] is the most effective graph-based ranking algorithm on the web which based on the number of incoming and outgoing links to find their ranking. The RTR of team $t$ is high if the team wins many matches against opposite teams that have a higher team strength and win-loss ratio. The RTR determined by using (6). 
$R T R(T)=\frac{1-d}{N}+d\left[\frac{R T R(T i)}{\mathrm{CTi}} * R T_{\text {Strength }}\right.$ ti $^{N}+, \ldots,+\frac{R T R(T n)}{C T n} *$
$\left.R T_{\text {Strength }}{ }_{t n}\right]$

Where,

- $\operatorname{RTR}(T)$ is the Region-wise TeamRank (RTR) of team $\mathrm{T}$.

- $\operatorname{RTR}\left(\mathrm{T}_{\mathrm{i}}\right)$ is the region-wise team rank of Team $\mathrm{T}_{\mathrm{i}}$ which links (lost matches) to team $\mathrm{T}$.

- $\mathrm{CT}_{\mathrm{i}}$ is the matches lost by team $\mathrm{T}_{\mathrm{i}}$.

- $\left(R T_{\text {Strength }}^{r} i^{r}\right.$ is the region-wise team strength (calculated from equation 2) of Team $\mathrm{T}_{\mathrm{i}}$ in region $\mathrm{r}$.

- While $\mathrm{d}$ is a damping factor which is usually set between 0 and 1 (in our experiment we set $d=0.85$ ).

- And $\mathrm{N}$ is the total number of teams played in region $\mathrm{r}$.

The in-links and out-links show won and lost matches by Team $t_{i}$ in region $r$. The network of teams created in Fig. 2, which played head-to-head matches against each other. The nodes represent the teams, and edges between them are the matches played. If two teams $t_{i}$, and $t_{j}$, played a match in a specific region, and if team $t_{i}$ wins from team $t_{j}$ then, $a$ directed edge is generated from team $t_{j}$ to team $t_{i}$. Consequently, a directed graph is generated iteratively to rank the teams by considering the number of matches played and the team strengths across the region.

3) Region-wise Weighted Team Rank (RWTR): In the Region-wise Weighted Team Rank (RWTR), region-wise win/loss ratio of a team $\mathrm{Ti}$ and region-wise win and lost matches through runs and wickets considered. A team that wins matches from another team with less number of runs and wickets ranked lower as compared to a team that wins matches with more number of runs and wickets across the region.

TABLE I shows how two basic parameters (runs and wickets) influence the region-wise ranking of a team $\mathrm{T}$ ? Assume team A and B have played 20 matches each in Asia region in which, both have won the same number of matches. If the sum of runs of the won matches of team $\mathrm{A}$ is 250 , and the sum of wickets of the won matches is 50. And the sum of runs of the won matches of team B is 400 and the sum of wickets of the won matches is 30 , then both teams, A and B, have different rank scores. In the above example, if we consider the win/loss ratio of each team then both teams have the same rank score. But when we consider runs and wickets through which a team won matches, then both teams have different rank scores 21.3 and 20.9, respectively as shown in TABLE II. Region-wise, WTR is calculated by using (7).

Where 10, 250 and 50 respectively are the number of matches, runs, and wickets through which Team A has won matches. TABLE III shows win and loss matches with runs and wickets.
$\operatorname{RWTR}(\mathrm{T})=\frac{1-d}{N}+\sum_{i=1}^{n} \frac{\left(R W T R\left(T_{\mathrm{i}}\right)\right.}{\mathrm{CTi}} * R T_{\text {Strength }}{ }_{t i}^{r}$

Where RWTR(T) is the Region-wise Weighted Team Rank of Team T in region r. RWTR $\left(T_{i}\right)$ is the Region-wise TeamRank of teams Ti which has lost matches to Team A.

$\mathrm{WC}\left(\mathrm{T}_{\mathrm{i}}\right)$ is the Region-wise out-links (lost matches) of Team $\mathrm{T}_{\mathrm{i}} \cdot R T_{\text {Strength }}{ }_{t i}$ (Calculated from (2)) is the Regionwise strength of team $T_{i}$ in region $r$. Region-wise Weighted out-links (RWO) calculated by using weighted arithmetic mean in (8).

$R W O=\frac{60(\text { Region }- \text { wise }- \text { winloss })+20(\text { Runs })+20(\text { wickets })}{\text { Region }- \text { wise }- \text { winloss }+ \text { Runs }+ \text { Wickets }}$

One can try different weights for the three features $33 \%$ w/l, $33 \%$ runs, $33 \%$ wickets or $40 \% \mathrm{w} / 1,30 \%$ runs, $30 \%$ wickets, but in our case, we used 60, 20 and 20 weights to each w/l, runs and wickets respectively.

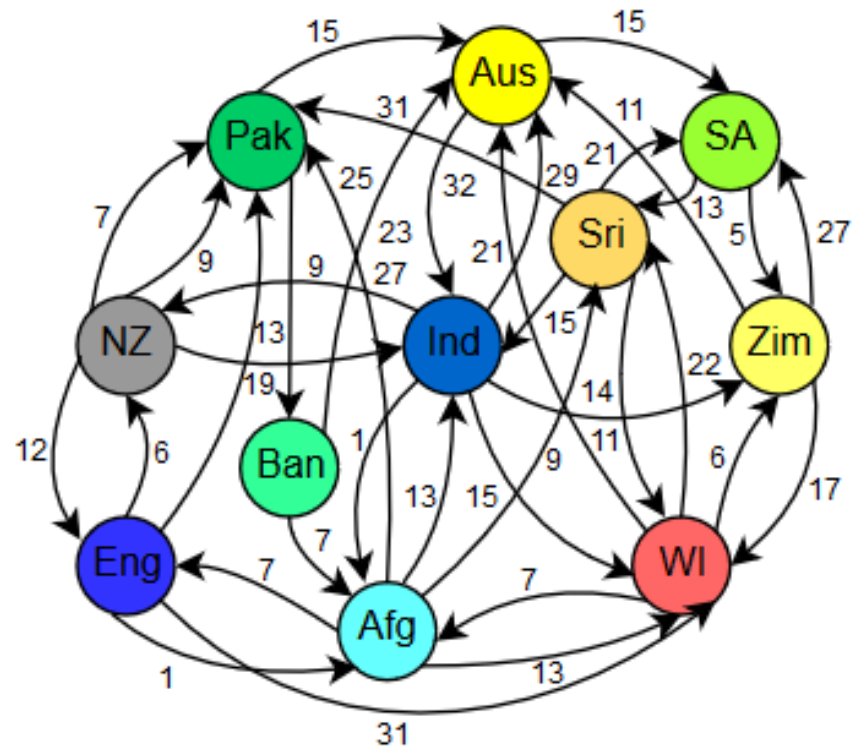

Fig. 2. A Graphical Representation of ODI Cricket Teams.

TABLE. I. RANK SCORES WHEN BOTH RUNS AND WiCKETS ARE IGNORED

\begin{tabular}{|l|l|l|l|l|l|}
\hline Teams & Win & Lost & W/L & Win by runs & Win by wicket \\
\hline A & 10 & 10 & 1.0 & 250 & 50 \\
\hline B & 10 & 10 & 1.0 & 400 & 30 \\
\hline
\end{tabular}

TABLE. II. RUNS AND WICKETS ARE CONSIDERED ALONG WITH W/L RATIOS

\begin{tabular}{|l|l|}
\hline Team A & Team B \\
\hline$\frac{60(10)+20(250)+20(50)}{(10+250+50)}=21.3$ & $\frac{60(10)+20(400)+20(30)}{(10+400+30)}=\mathbf{2 0 . 9}$ \\
\hline
\end{tabular}


TABLE. III. WON AND LOST MATCH'S RUNS AND WICKETS ARE CONSIDERED

\begin{tabular}{|c|c|}
\hline Team A & Team B \\
\hline$\frac{\frac{60(10)+20(250)+20(50)}{(10+250+50)}}{\frac{60(10)+20(400)+20(30)}{(10+400+30)}}=1.018$ & $\frac{\frac{60(10)+20(400)+20(30)}{(10+400+30)}}{\frac{60(10)+20(250)+20(50)}{(10+250+50)}}=\mathbf{0 . 0 9 8 2}$ \\
\hline
\end{tabular}

\section{EXPERIMENTS}

This section explains the dataset and performance evaluation in detail. It also provides a comparison of the ICC official teams ranking versus the region-wise ranking.

\section{A. Dataset}

The dataset is taken from the ESPNcricinfo website ${ }^{3}$ of One Day International matches played between January 2001 and August 2019. The ICC grouped cricket teams into three categories i.e., full member countries, associate member countries, and affiliate member countries ${ }^{2}$. In our case, we considered only full member countries; they have the right to play Twenty20, One-Day, and Test cricket matches and follow the ICC rules. The authors considered only those teams in the domain of ranking which had played at least ten ODI matches across the region.

\section{B. Results and Discussions}

The proposed ranking determined the strengths and weaknesses of a team across the region. If a team visits to play a match against the opponent team, having a regional ranking enables a more equitable regional distribution. The captain, the coach, and the team's management should be aware of their teams' strength against the opposite team from the visiting region so that the selectors select a strong combination from his team.

There is no statistical test to say which ranking is better; however, Spearman's Rank Correlation can be used to assess the reliability and validity of the proposed ranking methods. The region-based ranking score of all the regions of RTR and RWTR, respectively, as shown in TABLE VII and computed the Unified Region-based Team Rank (URTR) and the Unified Region-based Weighted Team Rank (URWTR) respectively. Besides, the URTR and URWTR scores are merged and computed the UTR, as shown in TABLE VIII. Consequently, a non-parametric correlation (Spearman rho) is used to assess the validity and reliability of the proposed (URTR, URWTR, and UTR) ranking as in TABLE V, and TABLE VI respectively with the ICC official ranting system using Spearman rho which had a strong correlation with the proposed methods. The results and discussions prove that the proposed methods are highly correlated and very useful in terms of ranking cricket teams across the region. The ICC only presents a general ranking based on the most recent performance of the teams. However, an ODI rating system from the ICC is used as a baseline for comparing the regionwise and a unified team's ranking. In addition, the outcomes of the proposed methods are also compared subjectively with the existing ICC ranking.
TABLE. IV. ICC CURRENT RANKING FOR ODI CRICKET TEAMS

\begin{tabular}{|l|l|l|l|l|}
\hline Teams & Matches & Points & Ratings & Rank \\
\hline England & 54 & 6,745 & 125 & 1 \\
\hline India & 58 & 7,071 & 122 & 2 \\
\hline New Zealand & 43 & 4,837 & 112 & 3 \\
\hline Australia & 50 & 5,543 & 111 & 4 \\
\hline South Africa & 47 & 5,193 & 110 & 5 \\
\hline Pakistan & 49 & 4,756 & 97 & 6 \\
\hline Bangladesh & 46 & 3,963 & 86 & 7 \\
\hline Sri Lanka & 54 & 4,425 & 82 & 8 \\
\hline West Indies & 49 & 3,740 & 76 & 9 \\
\hline Afghanistan & 40 & 2,359 & 59 & 10 \\
\hline Zimbabwe & 35 & 1,538 & 44 & 12 \\
\hline
\end{tabular}

TABLE VII shows the ranking of the full member countries played ODI cricket matches across Asia, Africa, the Americas, Europe, and Oceania regions. The ICC team ranking is shown in TABLE IV, while the proposed ranking shown in TABLE VII. England, India, and New Zealand are the top 3 teams in the ICC ranking, while in the RTR ranking India, Pakistan, and Sri Lanka are the respective top three teams. Because of their more considerable team strengths and win-loss ratios compared to the other teams in the Asian region. Australia, Pakistan, and India are the respective top 3 teams in the RWTR method. Australian team ranks first because of winning matches with a higher number of runs and wickets.

In the ICC ranking TABLE IV, the top 3 teams are England, India and New Zealand while in the RTR ranking South Africa, Australia and Indian cricket teams are the first, second and third respectively because of their more considerable team strengths and win-loss ratios compared to the other teams in the Africa region. South Africa, Sri Lanka, and Australian cricket teams are the respective top 3 teams in the RWTR method. South African team ranks first because of winning matches with a higher number of runs and wickets. In the ICC ranking, the top 3 teams are England, India, and New Zealand while in the RTR ranking Australia, West Indies, and South African cricket teams are the first, $2^{\text {nd }}$ and $3^{\text {rd }}$ respectively because of their greater team strengths and winloss ratios compared to the other teams in the America region. West Indies, South Africa, and Australian teams are the respective top 3 teams in the RWTR method. West Indies team ranks first because of winning matches with a greater number of runs and wickets.

England, India, and New Zealand cricket teams are the top-3 teams in the ICC ranking while first, fourth, and third in the RTR ranking. Because of their higher team strengths and win-loss ratios compared to the other teams in the Europe region.

England, Australia, and Indian cricket teams are the respective top-3 teams in the RWTR method. England team ranks first because of winning matches with a higher number of runs and wickets.

${ }^{3}$ http://www.espncricinfo.com 
TABLE. V. CORRELATIONS OF THE ICC RANKING WITH URTR AND URWTR RANKING AND URTR WITH URWTR RANKING USING SPEARMAN'S RHO

\begin{tabular}{|c|c|c|c|c|c|c|}
\hline Teams & ICC Rank & URTR Rank & $\begin{array}{l}\text { URWTR } \\
\text { Rank }\end{array}$ & $\begin{array}{l}\text { Correlation between ICC } \\
\text { and URTR }\end{array}$ & $\begin{array}{l}\text { Correlation between ICC } \\
\text { and URWTR }\end{array}$ & $\begin{array}{l}\text { Correlation between URTR } \\
\text { and URWTR }\end{array}$ \\
\hline England & 1 & 6 & 7 & \multirow{11}{*}{$75.45 \%$} & \multirow{11}{*}{$61 \%$} & \multirow{11}{*}{$86.36 \%$} \\
\hline India & 2 & 3 & 2 & & & \\
\hline New Zealand & 3 & 2 & 6 & & & \\
\hline Australia & 4 & 1 & 1 & & & \\
\hline South Africa & 5 & 4 & 3 & & & \\
\hline Pakistan & 6 & 7 & 5 & & & \\
\hline Bangladesh & 7 & 9 & 10 & & & \\
\hline Sri Lanka & 8 & 5 & 4 & & & \\
\hline West Indies & 9 & 8 & 8 & & & \\
\hline Afghanistan & 10 & 11 & 9 & & & \\
\hline Zimbabwe & 11 & 10 & 11 & & & \\
\hline
\end{tabular}

TABLE. VI. CORRELATION OF ICC RANKING WITH UTR RANKING USING SPEARMAN’S RHO

\begin{tabular}{|c|c|c|c|}
\hline Teams & ICC Rank & UTR Rank & Correlation between ICC and UTR Ranking \\
\hline England & 1 & 7 & \multirow{11}{*}{$61 \%$} \\
\hline India & 2 & 2 & \\
\hline New Zealand & 3 & 6 & \\
\hline Australia & 4 & 1 & \\
\hline South Africa & 5 & 3 & \\
\hline Pakistan & 6 & 5 & \\
\hline Bangladesh & 7 & 10 & \\
\hline Sri Lanka & 8 & 4 & \\
\hline West Indies & 9 & 8 & \\
\hline Afghanistan & 10 & 9 & \\
\hline Zimbabwe & 11 & 11 & \\
\hline
\end{tabular}

TABLE. VII. REgION-WiSE RANKING FOR ONE-DAY INTERNATIONAL (ODI) CRICKET TEAMS

\begin{tabular}{|c|c|c|c|c|c|c|c|c|c|c|}
\hline \multirow{2}{*}{ Teams } & \multicolumn{2}{|c|}{ Asia Region } & \multicolumn{2}{|c|}{ Africa Region } & \multicolumn{2}{|c|}{ America Region } & \multicolumn{2}{|c|}{ Europe Region } & \multicolumn{2}{|c|}{ Oceania Region } \\
\hline & RTR & RWTR & RTR & RWTR & RTR & RWTR & RTR & RWTR & RTR & RWTR \\
\hline Pakistan & 0.033 & 0.74696 & 0.062 & 0.40362 & 0.0796 & 0.13616 & 0.060 & 0.17726 & 0.035 & 0.16214 \\
\hline India & 0.034 & 0.72251 & 0.063 & 0.45770 & 0.0793 & 0.17120 & 0.061 & 0.25561 & 0.049 & 0.39800 \\
\hline Sri Lanka & 0.032 & 0.71932 & 0.061 & 0.49659 & 0.0806 & 0.16141 & 0.058 & 0.15754 & 0.041 & 0.17313 \\
\hline Bangladesh & 0.023 & 0.29832 & 0.038 & 0.04464 & 0.0759 & 0.10898 & 0.053 & 0.06956 & 0.029 & 0.07260 \\
\hline Australia & 0.031 & 0.91622 & 0.064 & 0.48430 & 0.0872 & 0.27824 & 0.063 & 0.25479 & 0.063 & 0.70225 \\
\hline England & 0.029 & 0.48493 & 0.056 & 0.32687 & 0.0789 & 0.12784 & 0.065 & 0.36283 & 0.042 & 0.17805 \\
\hline New Zealand & 0.027 & 0.34328 & 0.060 & 0.35056 & 0.0794 & 0.12444 & 0.062 & 0.20638 & 0.062 & 0.56022 \\
\hline South Africa & 0.028 & 0.43838 & 0.066 & 0.69583 & 0.0831 & 0.31941 & 0.057 & 0.16441 & 0.047 & 0.18375 \\
\hline West Indies & 0.024 & 0.31721 & 0.047 & 0.12478 & 0.0839 & 0.45803 & 0.059 & 0.18169 & 0.046 & 0.20473 \\
\hline Zimbabwe & 0.021 & 0.19635 & 0.041 & 0.12539 & 0.0732 & 0.01947 & 0.051 & 0.03639 & 0.020 & 0.05000 \\
\hline Afghanistan & 0.026 & 0.51987 & 0.043 & 0.17877 & N/A & N/A & 0.031 & 0.02453 & N/A & N/A \\
\hline
\end{tabular}


TABLE. VIII. A UNIFIED TEAMS RANKING FOR ODI CRICKET TEAMS

\begin{tabular}{|l|l|l|l|}
\hline Teams & URTR Ranking & URWTR Ranking & UTR Ranking \\
\hline Pakistan & 0.05392 & 0.325228 & 0.189574 \\
\hline India & 0.05726 & 0.401004 & 0.229132 \\
\hline Sri Lanka & 0.05452 & 0.341598 & 0.198059 \\
\hline Bangladesh & 0.04378 & 0.118820 & 0.081300 \\
\hline Australia & 0.06164 & 0.527160 & 0.294400 \\
\hline England & 0.05418 & 0.296104 & 0.175142 \\
\hline New Zealand & 0.05808 & 0.316976 & 0.187528 \\
\hline South Africa & 0.05622 & 0.360356 & 0.208288 \\
\hline West Indies & 0.05198 & 0.257288 & 0.154634 \\
\hline Zimbabwe & 0.04124 & 0.085520 & 0.063380 \\
\hline Afghanistan & 0.03333 & 0.241057 & 0.137195 \\
\hline
\end{tabular}

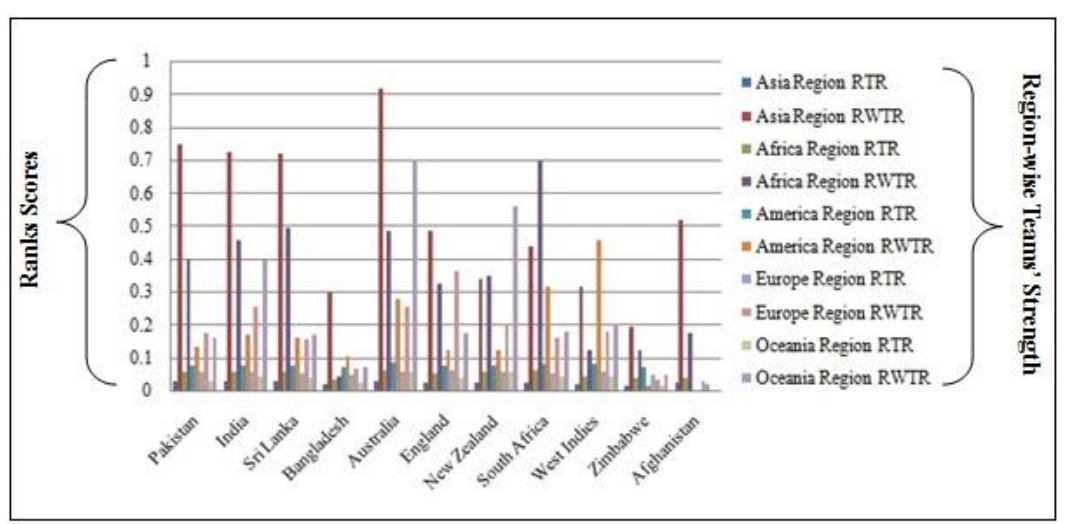

Fig. 3. Graphical Representation of Region-Wise Ranking for ODI Cricket Teams.

In the Oceania region, Australia, New Zealand, and India are the top-3 teams considering the RTR method while England, India, and New Zealand are the top-3 teams considering the ICC ranking as shown in TABLE IV. Because of their higher team strengths and win-loss ratios compared to the other teams across the region. Australia, New Zealand, and Indian teams are the respective top 3 teams in the RWTR method. Australian team ranks first because of winning matches with a higher number of runs and wickets. There are ups and downs between ODI cricket teams because of the teams 'strengths and weaknesses across the regions. Fig. 3 shows the graphical representation of region-wise teams' ranking for ODI cricket teams. It is clearly shown from the Fig. 3 that Australian cricket team is the highly ranked team in most of the regions.

The Unified Region-wise Team Rank (URTR) and the Unified Region-wise Weighted Team Rank (URWTR) are the weighted average scores of all the regions. Similarly, the Unified Team Rank (UTR) is the weighted average rank score of both the URTI and URTR methods. The Australian cricket team is ever first in the UTR while Indian and South African teams are second and third, respectively. The overall strength of the Australian team is higher as compared to the other teams. When the UTR results compared to the ICC rank, there is a strong correlation in the outcomes, as shown in TABLE VI.

The graphical representation of URTR, URWTR, and UTR ranking for ODI cricket teams is shown in Fig. 4. The Australian cricket team has overall better ranking in all the methods.

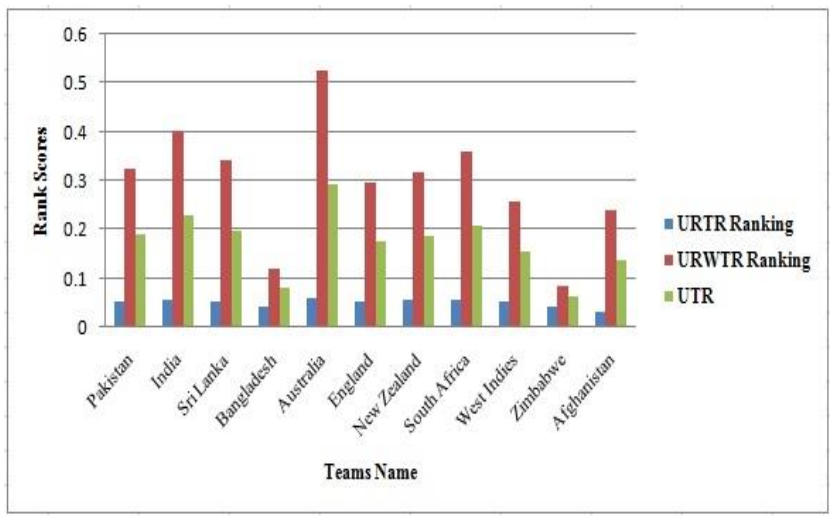

Fig. 4. Graphical Representation of a Unified Teams Ranking. 


\section{CONCLUSIONS AND FUTURE WORK}

We studied the ranking of ODI cricket teams across the region. We applied two methods; (1) Region-wise TeamRank (RTR) and (2) Region-wise Weighted TeamRank (RWTR). We investigated that the number of matches a team wins with the addition of team strength from the stronger team is very important by applying the TeamRank method. Furthermore, we concluded that the win/loss ratio and the number of runs and wickets through which a team wins from a team across the region also play an important role in cricket ranking. This paper is the first attempt to find out ranking cricket teams in ODI format to see the strength and weaknesses of each team across the region. In the future, we aim to build a deep model using Principal Component Analysis (PCA) for region-based teams' strengths and weaknesses to get better results and then compare the model with the traditional system.

\section{ACKNOWLEDGMENT}

This work was supported by the National Natural Science Foundation of China (61872261).

\section{REFERENCES}

[1] Borooah and J. Mangan, "The Bradman Class": An Exploration of Some Issues in the Evaluation of Batsmen for Test Matches , 1877-2006," no. June 2014, 2010.

[2] F. Ahmed, K. Deb, and A. Jindal, "Evolutionary Multi-Objective Optimization and Decision Making Approaches to Cricket Team Selection," pp. 1-23.

[3] T. Pagerank, C. Ranking, and B. Order, "The PageRank Citation Ranking: Bringing Order to the Web," pp. 1-17, 1998.
[4] P. Shah, "New performance measure in Cricket," vol. 4, no. 3, pp. 28 30, 2017.

[5] S. Mukherjee, "Quantifying individual performance in Cricket - A network analysis of Batsmen and Bowlers," Phys. A Stat. Mech. its Appl., no. 393, pp. 624-637, 2014.

[6] P. Taylor and H. H. Lemmer, "Predicting the names of the best teams after the knock-out phase of a cricket series," Eur. J. Sport Sci., no. September, pp. 37-41, 2014.

[7] P. Shah, "Measuring Fielding Performance in Cricket," vol. 23, no. 2, pp. 113-114, 2016.

[8] R. P. Bunker and F. Thabtah, "Applied Computing and Informatics A machine learning framework for sport result prediction," Appl. Comput. Informatics, 2017.

[9] M. Bailey and S. R. Clarke, "Predicting the match outcome in one day international cricket matches, while the game is in progress.," J. Sports Sci. Med., vol. 5, no. 4, pp. 480-487, 2006.

[10] T. B. Swartz, P. S. Gill, and S. Muthukumarana, "Modelling and simulation for one-day cricket," vol. 37, no. 2, pp. 143-160, 2009.

[11] D. Bhattacharjee and D. G. Pahinkar, "Analysis of Performance of Bowlers using Combined Bowling Rate," vol. 06, no. 03, pp. 184-192, 2012.

[12] A. Daud, F. Muhammad, H. Dawood, and H. Dawood, "Ranking Cricket Teams," pp. 1-17, 1998.

[13] H. Daud, A., Hussain, A., Abbasi, R. A., Aljohani, N. R., Amjad, T., \& Dawood, "Region-wise Ranking of Sports Players based on Link Fusion," in Companion of the web conference 2018, 2018, no. 1, pp. 259-266.

[14] W. Xing and A. Ghorbani, "Weighted PageRank Algorithm," in 2nd Annual Conference on Communication Networks and Services Research (CNSR 2004), 19-21 May 2004, Fredericton, N.B. Canada., 2004.

[15] S. Mukherjee and B. Engineering, "Identifying the greatest team and captain - A complex network approach to cricket matches," vol. 391, no. 23, 2012. 